\section{Analysis of variance of one-, two-, and three-treatment designs for a PDP-8*}

\author{
ROBERT BREAUX \\ Texas Tech University, Lubbock, Texas 79409
}

Operating instructions for a series of analysis of variance programs for one-, two-, and three-treatment experimental designs is described. The emphasis is on versatility, speed, accuracy, and sufficiency of output. The on-line aspect of FOCAL allows extensive transformations of raw data. Procedures and terminology conform to Kirk (1968) to provide information for pooling error terms for various models, mean comparisons, and trends analysis. Patches are given for data tape input.

This paper provides instructions for the use of a series of programs for analysis of variance with the PDP-8 family of computers. Some of the most often used experimental designs are factorial and split-plot analyses for one-, two-, and three-treatment experimental designs (Kirk, 1968). Unfortunately, it often requires as much time and effort to run these designs through a large computer (data transcription to cards, checking for accuracy, long turnaround times) as it does to perform the analysis with a calculator. Yet, one often-desires the arithmetic accuracy of the computer program. It was to combine the advantages of accuracy with the speed of a calculator, for small designs, that the programs described here were conceived. The emphasis is on versatility, ease of data input, sufficiency of information output, and speed of obtaining results with a minimum of hand calculations. Each program is written in the FOCAL programming language (DEC, 1970) and will run with a minimum of hardware: A Teletype/paper-tape reader and $4 \mathrm{~K}$ core for the one-way analysis programs and other small designs, $8 \mathrm{~K}$ for larger designs.

The statistical procedures and terminology described in this paper are taken from Kirk (1968). The user should familiarize himself with the use of "bracket terms" in particular. Since the source tables are for a mixed model (where treatments are fixed effects and Ss are random effects), bracket terms are necessary in pooling to find the proper error term for the other models. Kirk (1968) will provide a handy reference for further data analysis, including mean comparisons and trend analysis. For those unfamiliar with Kirk's notation, Table 1 lists the designs that can be analyzed using these programs, notations used by other authors, and

*Progiams are available from DECUS Program Library, 146 Main Street, Maynard, Massachusetts 01754 . variables stored. Likewise, the random-block designs calculate $\mathrm{S}$ the CRF analysis. interaction terms and will handle fewer levels per treatment than will completely randomized designs. For example, with $8 \mathrm{~K}$, the $\mathrm{CR} \cdot \mathrm{k}$ program handles at least 100 groups; the CRF-pqr is limited only by the number of levels of $r$ and $q$, i.e., the product rq can be as large as 60 . In both cases, increasing the number of Ss is of no consequence other than increasing the amount of time for data input.

In the second step of analysis, the program asks for the number of data points per line. This allows the user to format the input. A rule of thumb is to set this to the same value as the number of levels of the treatment that varies the fastest (explained later). One advantage of this procedure is that the printout is arranged such that each row is a complete cell.

In the third step of analysis, the data are input in a program-specific order. The process of data indexing is important here and refers to how each data point is labeled. Indexing is important since the program cannot distinguish one data point from another. The problem is solved by inputing the data in a particular order. Each program specifies the exact order. Table 2 shows the process of data indexing for a simple two-treatment design. Each data point $\left(x_{i j k}\right)$ is subscripted by the level of Treatment $A, B$, and the $S$ number under which it was recorded. For example, the data point $x_{123}$ was recorded under Level 1 of Treatment $A$, under Level 2 of Treatment B, and for S 3. The extension of this procedure to a three-treatment design, should be obvious; it is analogous to the summation notation used by many authors. As an example of indexing, note theprogram instructions for the CRF-pq design: Enter data, with $\mathrm{N}$

Table 1

Page Number References for Each Desion

\begin{tabular}{|c|c|c|}
\hline Kirk's Notation & Alternate Name & Page Number \\
\hline \multicolumn{3}{|l|}{ One Treatment } \\
\hline CR-k & $\begin{array}{l}\text { Completely Between } \\
\text { One-Way Analysis }\end{array}$ & 104 \\
\hline $\mathbf{R B}-\mathbf{k}$ & $\begin{array}{l}\text { Completely Within } \\
\text { One-Way Analy sis }\end{array}$ & 132 \\
\hline \multicolumn{3}{|l|}{ Two Treatments } \\
\hline CRF-pq & Completely Between & 173 \\
\hline CRH-p $(q) *$ & Nested Treatments & 229 \\
\hline RBF-pq & Completely Within & 237 \\
\hline SPF-p.q & Between/Within, or Split-Plot & 248 \\
\hline \multicolumn{3}{|l|}{ Three Treatments } \\
\hline CRF-pqr & Completely Between & 217 \\
\hline CRH-p(q)(r) & Two Nested & 234 \\
\hline CRH-p $(q) r$ & Crossed/Nested & 235 \\
\hline SPF-pr.q & Two Between/One Within & 283 \\
\hline $\begin{array}{l}\text { SPF-p.qr } \\
\text { RBF-pqr }\end{array}$ & One Between/T wo Within & 298 \\
\hline
\end{tabular}

*The source table for the CRH analysis may be calculated from the bracket terms of 
Table 2

Data Indexing

\begin{tabular}{llllll}
\hline & \multicolumn{2}{c}{$A_{1}$} & & \multicolumn{2}{c}{$A_{2}$} \\
\cline { 5 - 6 } & $B_{1}$ & $B_{2}$ & & $B_{1}$ & $B_{2}$ \\
\hline$s_{1}$ & $x_{112}$ & $x_{121}$ & & $x_{211}$ & $x_{221}$ \\
$s_{2}$ & $x_{122}$ & $x_{122}$ & & $x_{212}$ & $x_{222}$ \\
$s_{3}$ & $x_{123}$ & $x_{123}$ & & $x_{213}$ & $x_{223}$ \\
\hline
\end{tabular}

varying fastest and $A$ varying slowest This means that the order of data input is such that the $S$ index varies fastest, the Treatment $B$ index next, and the Treatment $A$ index varies most slowly. The order of data input is $x_{111}, x_{112}, x_{113}, x_{121}, x_{122}, x_{123}$, $x_{211}, x_{212}, x_{213}, x_{221}, x_{222}, x_{223}$. It is obvious that the $S$ index changes most often, then the index of Treatment $B$. The least often changing index is that of Treatment $A$.

Raw data are not stored by the program. Sums, sum of squares, and means are computed during data input. To conserve storage area, means are printed as they are calculated. This has the added feature of producing a printout that groups data cells and follows them immediately with the means of those cells.

Transformations require that the user have some knowledge of FOCAL; there are many functions available in the FocAL language for transformations, both trigonometric and algebraic. A transformation of raw data could, of course, be accomplished by hand prior to data analysis, but it is much easier to modify the data input statement so that the transformation (implying that $B$ varies in the middle).

occurs before summing and squaring takes place. This is done easily since FOCAL is an on-line language. The particular statement to be modified in each program is listed along with an example transformation in Table 3 . For example, if speed scores $(1 / \mathrm{sec})$ are desired and the measure taken is time, one need only modify statement 3.15 of the CR-k program to read: 3.15 A $X ; S X=1 / X$. Thus, the inverse (speed) of the raw data (time score) is calculated and analysis is performed upon that value, the speed score. In this way, the number of transformations available is quite extensive. If it were also desirable to list the transformed data, the change could be 3.15 A X;S X=1/X;T X. With the echo removed or if input were from the high-speed reader, only the speed score would appear on the printout.

A final point can be made for ease of data analysis. When transformations are anticipated or when more than one measure is taken (e.g., trial of last error, trials to criterion, latency), it may be useful to punch the data on paper tape before any analysis is done. For example, modification of the input statement can allow for ignoring all but latency scores by inserting two dummy variables before " $X$ " (see Table 3). A small hint may be in order at this point. With a little planning, the advanced assembly programmer can output his data on paper tape initially, and in this way eliminate the chance of human error at least for data transcription. Another advantage of paper tape is that it can be checked for

Table 3

Statements to Modify When Uting Transformations

\begin{tabular}{|c|c|c|c|}
\hline Desion & $\begin{array}{l}\text { State- } \\
\text { ment }\end{array}$ & $\begin{array}{c}\text { Example } \\
\text { Transformation }\end{array}$ & New Statement \\
\hline CR-k & $\mathbf{3 . 1 5}$ & $\begin{array}{l}\text { Inverse and High } \\
\text { Speed Reader Input }\end{array}$ & $3.15 * ; A X ; * ; S X=1 / X$ \\
\hline $\mathbf{R B}-\mathbf{k}$ & $\mathbf{3 . 1 5}$ & Square Root & 3.15 A X; $\mathrm{X}=\operatorname{FSQT}(\mathrm{X})$ \\
\hline CRF-pq & 4.04 & $\begin{array}{l}\text { Analyze Every } \\
\text { Other Input* }\end{array}$ & $4.05 A Y, X$ \\
\hline $\mathbf{R B F}-\mathbf{p q}$ & 4.05 & Sine & 4.05 A X; $\mathrm{S} \mathrm{X}=\operatorname{FSIN}(\mathrm{X})$ \\
\hline SPF-p.q & 15.15 & $\log _{e}$ & 15.15 A $X ; s ~ X=F L O G(X)$ \\
\hline $\begin{array}{l}\text { Three Treat- } \\
\text { ment Designs }\end{array}$ & 6.15 & Total Two Scores & $6.15 A Y, X ; S X=Y+X$ \\
\hline
\end{tabular}

- Note that $Y$ is a dummy variable and is never used in computations.
Table 4

Patches for Data Tape Input

\begin{tabular}{rccc}
$\begin{array}{c}\text { Loca- } \\
\text { tion }\end{array}$ & Contents & $\begin{array}{c}\text { Change } \\
\text { to }\end{array}$ & Effect \\
\hline 2163 & 4551 & 7000 & Remove Echo \\
63 & 2676 & 1354 & Turn Off \\
64 & 2666 & 2414 & Interrupt \\
2732 & 6001 & $\mathbf{5 3 3 6}$ & \\
2762 & 6046 & 7000 & \\
\hline
\end{tabular}

accuracy, then used repeatedly in each transformation analysis without concern for typing errors. To further aid input, some patches are provided by DEC (1971) and are listed in Table 4 to remove the interrupt and to remove the echo, so that input buffer overflow is eliminated for the low-speed paper-tape reader. If a high-speed reader is available, a simple statement modification is possible. An example for input from the high-speed reader is given in Table 3 for the CR-k design.

Some mention should be made concerning the accuracy of these programs. They were checked for errors by using the data in Kirk (1968). The discrepancies found were attributed to rounding errors.

In sum, instructions for the use of a set of programs that allow on-line data analysis with a minimum of effort, yet a maximum of versatility, have been presented. Unless transformations are required, little programming knowledge is required. The programs allow analysis of variance for the most popular one-, two-, and three-treatment designs, and include sufficient output to analyze various models, to carry out trend analysis, and to do mean comparisons.

REFERENCES

Digital Equipment Corporation, Programming Department, Software Writing Group. Programming languages. Mrynard, Mass: DEC Program Library, 1970.

Digital Equipment Corporation, Software Information service. Software performance summary for the PDP-8 and PDP-12. 2(9). Maynard, Mass: DEC Program Library, 1971.

KIRK, R. E. Experimental design: Procedures for the behovioral sciences. Belmont, Calif: Brooks/Cole, 1968. 